\title{
The Evolution of Three-Dimensional Cell \\ Cultures Towards Unimpeded Regenerative Medicine and Tissue Engineering
}

\author{
Aleksandar Evangelatov and Roumen Pankov \\ Additional information is available at the end of the chapter \\ http://dx.doi.org/10.5772/55564
}

\section{Introduction}

The idea that cellular survival and growth could be maintained outside the body was recognized as possible almost hundred years ago when the German zoologist Wilhelm Roux described a successful experiment where he cultured chick neural crest in warm saline for a few days [1]. Nobel Prize winner Alexis Carrel performed numerous experiments clearly showing that tissue explants, including connective tissue and heart tissue, could be cultured in vitro preserving their characteristics for prolonged periods of time [2] supporting the notion that entire organs could be cultured in vitro. A defined synthetic mixture of amino acids, salts, carbohydrates, vitamins and serum was shown to support cells in vitro[3], thus unifying a major variable in cell culturing experiments and providing a possibility for rapid development of this novel method. Since the establishment of the first cell line by Gey et al.[4] in 1951 cell culturing has become one of the most widely used methods with exceptional contribution to the advances in almost all fields of contemporary biology - cell biology, genetics, cell biochemistry, physiology etc. Significant progress in the field made possible numerous achievements that were believed to be the foundations of personalized medicine. Among these is the isolation of the first line of murine stem cells [5, 6] in 1981, followed by establishment of the first human embryonic stem cell lines by Thompson [7].

Current knowledge of cellular behavior is mainly acquired by studies concerning homogenous populations of cells cultured as monolayers. This simplified approach towards understanding the essence of the mechanisms, underlying the processes determining life and death of a cell has undoubtedly provided scientists with enormous amount of knowledge. However, recent advances in the field of three-dimensional cell cultures have revealed a lot of imperfections in 
this limiting approach. Growing fibroblasts on flat, rigid surface of the Petri dish or flask results in major differences in adhesion formation and maturation, proliferation, cell signaling, migration and cytoskeletal function, compared to the three-dimensional environment [8-12]. Even changes in stiffness of the two-dimensional (2D) substrate have shown significant difference in fibroblasts response to the environment [13, 14]. Being cultured on 2D surfaces, these non-polar cells are forced to adapt to polar setting, thus significantly changing their response to the environment. One could argue that this culturing method would be ideal for epithelial cells, allowing them to form distinct apical and basal layers. Nevertheless growth in 3D environment allows normal polarization and differentiation of epithelial cells [15] and is a prerequisite for the formation of duct-like structures in vitro [16]. Thereby the emerging differences between conventional, monolayer cell cultures and in vivo cellular behavior led to the increasing number of scientists aiming to provide in vivo-relevant results.

The idea of cell culturing in 3D environment is not a novel one though [17], but until recently the importance of the cell environment - its dimensionality, stiffness, elasticity, composition and remodeling during tissue morphogenesis and disease, remained neglected. Number of studies have shown that knockdown of extracellular matrix (ECM) components like fibronectin, collagen, laminin, aggrecan, etc. lead to lethal phenotypes or severe pathologies during embryonic or post-natal development [18-24]. Furthermore, recent discoveries like the importance of $\mathrm{Cdc} 42$ for the appropriate acquisition of apical and basal polarity during morphogenesis or cell specification during tubulogenesis wouldn't have been possible without the use of 3D model systems $[25,26]$. Thus the concept of the importance of the surrounding settings emerged and an entirely new scientific direction evolved, focusing on the role of the extracellular matrix and its importance in cell biology.

In the living organism cells are usually embedded in a complex three-dimensional extracellular matrix that is dynamic in its structure[27] and rarely do have the opportunity to attach to planar, rigid substrates. Reciprocal interactions between cells and the ECM facilitate signaling to and from cells and lead to continuous reorganization of the environment [10]. Investigation of the dynamics of the ECM, its structure in different tissues and cellular response to changes in the mechanical properties of the extracellular matrices have shown that cells are not only able to feel and respond to the environment, but also to cause changes to the environment's mechanical properties [14]. These interactions deliver further signals responsible for cell growth and differentiation, survival, migration and reorganization of the resident tissue [28]. In addition when one thinks of cells in the third dimension it has to be considered that cells are not just randomly incorporated in the ECM but form complex 3D structures, characteristic of the specific tissue or organ [29].

The recognition of the importance of dimensionality of cellular environment encouraged the creation of a variety of three-dimensional culturing systems. Essentially they could be divided into two groups - three-dimensional systems made of artificial materials, mimicking the natural components of the matrix and three-dimensional culturing systems based on natural ECM components, or cell-synthesized ECM. This article's focus will be mainly on the later 3D culturing models. 


\section{Artificially fabricated 3D scaffolds}

Design of artificial 3D environments for cells aims at creating an environment that would mimic the physical properties of the natural extracellular matrix along with the signaling cues it provides for cell development. Such scaffolds should be non-toxic, preferably biodegradable in time and must allow cell attachment and migration as well as diffusion of vital nutrients [30]. Cells should be able to proliferate and differentiate, and subsequently to synthesize and organize their own ECM. In time, eventually, the naturally synthesized and organized extracellular matrix should substitute the artificial scaffold. All those prerequisites mean that the surface should be suitable for cell attachment, have the adequate chemical and mechanical properties to support adhesion, proliferation and differentiation. To our knowledge there are currently several reported methods for fabricating appropriate artificial scaffolds:

- The technique of controlled rate freezing and lypohilization [31] produces suitable extracellular matrices, in spite of the relatively uneven pore sizes. Another drawback of this method is that the produced artificial matrix does not have fibrillar structure which is common for the natural ECM.

- Phase separation of nanofibers [32] yields fibrous matrices, but also produces pores with uneven sizes.

- Formation of porous scaffolds [33]. This method allows the formation of matrices with similar pore sizes, but is limited by the matrix thickness

- Computer-assisted design and manufacturing (CAD/CAM) technologies [34] allow the formation of geometrically complex structures, with precise control over pore sizes, variety of material choices, layering, etc. with the use of some biological materials. A drawback of these technologies is the lack of ability to produce fibrous structures in the nanoscale.

- Nanofiber self assembly of thinner fibrils [35] is a relatively expensive method, but allows the production of thinner than $10 \mathrm{~nm}$ filaments that subsequently form thicker fibers, organizing structures, similar to the natural extracellular matrix.

- The electrospinning technology [36] is used to produce matrices of fibers in the micro- and nanoscale by employment of numerous polymers including also natural polymers. Even though this technique allows for much more precise tailoring of the biological and mechanical properties of the resulting matrices, it is still limited by the achieved small pore sizes thus limiting cellular migration within the matrix.

All these methods find application in medicinal practice and tissue engineering, but there are still many questions to be answered regarding their safety with long term usage, biodegradability, their influence on cellular signaling, proliferation and differentiation, etc. that are beyond the scope of this review.

\section{3D culturing systems based on natural components of the ECM}

Gels present the easiest, most affordable and quickest way to provide cells with a threedimensional environment that can be further manipulated by variety of methods in order to 
modulate their properties. Different types of gels have been utilized during the past few decades with collagen, fibrin, hyaluronan and basement membrane extract (BME) gels being the most frequently employed.

\subsection{Collagen gels}

Collagens represent the most abundant type of proteins in mammals making up to $35 \%$ of the whole body protein content. They are found in fibrillar and non-fibrillar forms and are widely distributed among tissues with a distinct structural, organizational and density variance among tissues and organs. Currently, 28 types of collagen have been described that are known to be products of 49 different $\alpha$-chain gene products [37]. Collagen gels are one of the first employed 3D culturing systems used to study cellular adhesion and migration in threedimensional environment $[17,38]$. Currently collagen gels have found application in a wide variety of studies in the field of cellular motility [11] and the importance of physical parameters such as density, stiffness and elasticity for cellular adhesion, proliferation, migration and contraction [39]. Fibroblast cells, seeded in collagen gels, have been shown to acquire different morphology when compared to fibroblasts grown on two-dimensional surfaces and appear similar to their counterparts in normal connective tissue. The three-dimensional environment of the gel mimics the in vivo conditions and also causes normal polarization and differentiation of epithelial cells [16]. It has also been developed as a model for studying the progression of prostate and breast cancer $[40,41]$.

Few types of collagen gels are most widely employed as three-dimensional culturing systems. Pure gels that remain attached to the dish after polymerization are considered as "stressed". The cells plated within this 3D environment usually exert isometric tension. If the gels are released, detached from the bottom of the Petri dish, they contract and represent the so called "relaxed and loaded" gels. If the gels are detached from the dish before cells are being seeded, they represent "relaxed and unloaded" gels. The ease of manipulation of the collagen gel's stiffness provides a useful tool for the evaluation of the role of isometric tension for cell survival and differentiation as well as for the investigation of mechanical features of the environment and it's reorganization during wound healing processes or various pathological conditions [39]. Some interesting features of cell migration in 3D have been established using those different types of collagen gels. Migration in softer substrates, or compliant matrices, results in migratory activity that is independent of the small GTPase Rho [39], thus, the cell acquires an amoeboid phenotype. As tension increases and the extracellular matrix becomes more rigid, the migratory mode of fibroblasts switches over to Rho dependent migration [42] with a mesenchymal phenotype. Moreover, in order for fibroblasts to differentiate to myofibroblasts upon TGF $\beta$ stimulation, a typical process during wound healing, increased tension in the extracellular matrix is required [43]. Reorganization of the extracellular matrix also appears to be dependent on the mechanical properties of the substrate as incorporation of fibronectin in the extracellular matrix requires internal tension of the ECM [44].

Collagen gel-based dressings were also used in some of the first attempts to create in vitro equivalents of full thickness skin for regenerative medicine and tissue engineering applications 
[45]. Currently there are a few temporary and semi-permanent dressings available as off-theshelf products, intended for use as bioconstructs for skin reconstruction [46].

\subsection{Fibrin gels}

Fibrin gels are another type of easily manufactured 3D culturing systems. They are formed as a result of thrombin cleavage of fibrinogen, resulting in a mesh of fibrin fibers. Stressed, relaxed and loaded and relaxed and unloaded types of fibrin gels are also frequently used model systems. In normal physiological conditions fibrin represents the provisional matrix of the clot, formed after wounding. Thus, the defined use of fibrin gels to study cellular invasion and contraction during the processes of wound healing [47] has emerged. Historically, polymerized fibrin gels were one of the first scaffolds used for skin tissue engineering after severe burns or for treatment of chronic wounds [48]. Currently fibrin gels have found further applications in the field of vascular tissue engineering and are being extensively studied especially as a possible resolution of a number of rapidly growing problems related to arterial occlusive diseases [49]. Studies in cardiac tissue engineering and cartilage regeneration and reconstruction have also benefited of the use of this natural, biodegradable scaffold [50,51].

\subsection{Hyaluronan gels}

Hyaluronan or hyaluronic acid is widely distributed throughout all tissues glycosaminoglycan. It is an anionic, nonsulfated polysaccharide, formed on the plasma membrane instead of in the Golgi apparatus, with molecular weight varying between $5 \mathrm{kDa}$ and 2,000 kDa. Hyaluronan is a major component of the cartilage, synovial fluid, the extracellular matrix of the skin and has a major role during the developmental processes [52]. Presumably, due to the high abundance of the molecule and its role during development, wound healing and migration, hyaluronan gels became another suitable three-dimensional model for studies in numerous fields.

As a natural component of the cartilage, hyaluronan is being used as a milieu for culturing chondrocytes in vitro. Current research indicates that culturing in tissue-like hyaluronic 3D environment sustains chondrocytes phenotype, leading to increased proliferation, sulphated glycosaminoglycans production as well as collagen type II and aggrecan synthesis and indeed supports chondrogenic differentiation [53]. Additional studies have shown promising results concerning the use of hyaluronan for cartilage repair and as scaffold for regenerative medicine [54-56]. Another intriguing direction of studies is the utilization of three-dimensional hyaluronic acid scaffolds for culturing mesenchymal stem cells. Promising results from culturing stem cells in three-dimensional hyaluronan-based scaffolds have been obtained in regard to the generation of cartilage-like tissue for the use of regenerative medicine [57]. Hyaluronic acid gels have been also investigated as possible 3D scaffolds for culturing cardiomyocytes and hepatocytes in vitro $[58,59]$. Therapeutical use of hyaluronan gels has been reported also in the field of adipose tissue engineering [60] as well as in neuromedicine. The use of this biodegradable material has revealed promising results in cell and drug delivery to the central nervous system[61]. 


\subsection{Basement membrane extract gels}

Almost 30 years ago the Engelbreth-Holm-Swarm (EHS) mouse sarcoma cell line was found to secrete vast amount of unknown protein mixture. Later it was determined that this mixture was composed of the typical for the basement membrane proteins collagen IV, laminin, entactin and the heparansulfate proteoglycan [62]. Further components like matrix metalloproteinases as well as number of growth factors were also identified in this complex mixture. This protein composite is marketed by BD Biosciences under the trade name Matrigel $®$, but similar products are available from other sources. Matrigel resembles the complex extracellular matrix found in many tissues and is thus used by cell biologists as a substrate for cell culturing.

Utilization of the basement membrane matrix led to numerous scientific discoveries. For the first time the EHS matrix was used as a substrate to cultivate Sertoli cells, which led to their survival and the differentiation of the accompanying germ cells[63]. The first in vitro myelination was also observed in cultures based on the basement membrane matrix. Carey et al. demonstrated in 1986 that rat Schwann cells cultured in 3D conditions based on basement membrane matrix show increased dendrite outgrowth and myelination [64]. Thus a reliable in vitro model for investigation of nerve regeneration was established.

Another interesting observation was made when epithelial and endothelial cells were cultured on basement membrane matrix. Both cell types show different morphology compared to flat 2D surfaces, but also form specific structures depending on whether cells are cultured on top or within the matrix. Ducts were formed when epithelial cells were cultured on top of the matrix, and acinar-like structures appeared when cells were embedded within the BME gel $[65,66]$. Different types of acinar epithelial cells (breast epithelial cells, salivary gland cells, pancreatic and prostate cells) form distinct structures in 3D indeed clearly supporting the importance of the extracellular matrix in cellular differentiation and proliferation. Endothelial cells form capillary-like structures when seeded in lower counts, or monolayers when seeded in higher counts. These observations made possible the development of in vitro models for studying angiogenesis. Tumor-induced angiogenesis is a key prerequisite for neoplastic progression thus angiogenesis suppression is one of the major directions investigated as possible cancer treatment. Vascular endothelial cells form capillary-like structures when plated on 3D basement membrane gels and have provided a suitable model for testing pharmacological substances and screening of chemical agents as angiogenic inhibitors [67].

Basement membrane extract gels provided also another important model system aimed at investigation of cancer invasion and metastasis. Kramer et al. noticed that normal, nonmalignant cells polarize on top of the matrix whereas malignant cells invaded the 3D basement membrane [68]. While normal cells attached, polarized and differentiated on top of the matrix and showed almost no migratory tendency, malignant cells exhibited increased invasive and migratory phenotype within the matrix. Formation of long protrusions in the direction of migration and channels of degraded matrix behind the cells were observed. Evidently, malignant cells mimicked their in vivo invasive behaviour since synthesis of proteolitic enzymes and degradation of the basement membrane are key events during tumor metastasis. Continuous investigation led to the development and improvement of invasion and metastasis 
assays $[69,70]$ providing identical conditions and criteria to measure the invasiveness of tumors and the efficiency of treatment.

Maybe one of the most significant discoveries related to the progress of regenerative medicine and tissue engineering is the possibility to culture stem cells on basement membrane matrix [71]. Normally the basement membrane is the first ECM to be synthesized in the developing embryo [72] hence the logical use for culturing stem cells. Feeder layers of irradiated mouse embryonic fibroblasts were required for long term culturing and limited the large scale production of human embryonic stem cells. Moreover, they present the possibility of viral cross contaminations. Utilization of other extracellular matrix components like fibronectin, collagen I, collagen IV or artificial substrates has failed to support undifferentiated stem cells growth [73], whereas coating of the dish with BME extract retained the undifferentiated state of human embryonic stem cells for up to 30 passages, maintaining their proliferation rate, high telomerase activity, normal karyotype and the expression of the pluripotency markers.

\subsection{Cell-derived matrices}

The use of three-dimensional gels of collagen, fibrin, basement membrane matrix or glycosaminoglycans represents a significant advance in cell culturing. Those gels provide the much needed dimensionality to bring the environmental conditions closer to the in vivo settings. A major disadvantage of those models though is the fact that they still lack the chemical complexity and spatial organization of the ECM, characteristic for tissues (Figure 1). Indeed cells plated in three-dimensional gels degrade and reorganize to some extent the constituents of their surroundings and also secrete and integrate new components within the existing extracellular matrix [74]. Presumably cells are trying to shape their environment by their liking, but all those processes are believed to keep cells in an "activated" state that is unnatural for healthy tissue. In the quest for creating better model systems that approximate closer to different healthy tissues a few groups have developed during the last few decades affordable methods for creating cell derived three-dimensional cultures and matrices [9, 75-79]. Those methods are based on in vitro preparation of three-dimensional extracellular matrices made by the cells themselves. The result is a naturally synthesized and organized extracellular matrix, providing in vivo-like conditions.

Conducted research, based on these tissue-like cultures has already demonstrated significant differences between cells grown in three-dimensional cell derived matrices and other 3D culturing methods. Ahlfors and Billiar [79] demonstrated that culturing fibroblasts in appropriate conditions induces synthesis of extracellular matrix components, thus resulting in a multilayer culture, with mechanical properties approximating normal tissue. Investigation of the mechanical properties of these cell derived 3D cultures revealed that the resulting matrices are stronger than collagen or fibrin 3D cultures. Moreover cells in naturally synthesized cultures had higher protein synthesis rate than fibroblasts cultured in collagen or fibrin gels, and more importantly, inhibition of collagen synthesis was not observed at later stages of cultivation. The production of matrices was achieved in serum supplemented media as well as in chemically defined media. The second option makes exploitation of this method relatively 


\begin{tabular}{|c|c|c|c|}
\hline $\begin{array}{c}\text { THREE } \\
\text { DIMENSIONALITY }\end{array}$ & $\begin{array}{l}\text { MECHANICAL } \\
\text { PROPERTIES }\end{array}$ & $\begin{array}{l}\text { CHEMICAL } \\
\text { COMPLEXITY }\end{array}$ & $\begin{array}{c}\text { NATIVE } \\
\text { ORGANIZATION }\end{array}$ \\
\hline \multicolumn{4}{|c|}{ Artificially fabricated 3D scaffolds } \\
\hline \multicolumn{4}{|c|}{ 3D gels of natural ECM components } \\
\hline \multicolumn{4}{|c|}{ Cell-derived 3D matrices } \\
\hline \multicolumn{4}{|c|}{ Decellularized tissue and organ scafolds } \\
\hline
\end{tabular}

Figure 1. Schematic presentation of the main characteristics of 3D cell culture systems. While artificially fabricated 3D scaffolds can be designed with specific three dimensional organization and desired mechanical properties, they, however, generally lack the chemical complexity of the natural ECM. This limitation can be compensated to a certain extent by incorporation of natural ECM proteins or their peptides (indicated by additional small arrow). 3D gels, made out of natural ECM components have also restricted chemical complexity and in addition their structural organization is a result of spontaneous, rather than cell-directed polymerization. Cell-derived 3D matrices and decellularized tissues and organs meet the general requirements for in vivo-like 3D environment. However, they often pose difficulties in preparation and restrictions in their mass production (see text for details).

expensive, but increases the chances of utilization of such cultures for the needs of tissue engineering by greatly diminishing the possibilities of species or viral cross contamination.

As we have previously discussed, cellular morphology of fibroblasts differs between 2D and 3D conditions. Even though fibroblasts appear to acquire the same morphology when plated on collagen gels and cell derived matrices, there are a number of issues to be considered fibroblasts attach, proliferate and migrate at much higher rate in cell derived matrices compared to collagen gels [9]. Though dimensionality influences fibroblast morphology one must also take into account the heterogeneity and specific organization of the extracellular matrix which are of significant importance as well. The fact that 3D matrix adhesions were established in cell derived extracellular matrices, but not in fibrin or collagen gels, or 2D surfaces coated with ECM components [9] supports the notion of the importance of structural organization of the environment. Both, the components and their colocalization in these adhesion structures differ from focal and fibrillar adhesions. Formation of such in vivo-like adhesions appears to depend on the heterogeneity and organization of the ECM and their formation does not require de novo protein synthesis during cellular adhesion. Major differences in signal transduction of fibroblasts in cell derived matrices were also discovered. The focal adhesion kinase (FAK), a molecule with exceptionally important role for cellular adhesion in conventional monolayer cultures, appears to be bypassed in in vivo-like conditions [8, 9]. In spite of this, downstream molecules maintain or even augment their activities, resulting in increased proliferation for example. Furthermore culturing of fibroblasts as a cell derived, three-dimensional multilayer culture leads to altered distribution of plasma membrane lipids 
[80-82]. Changes of the localization and properties of plasma membrane cholesterol and sphingomyelin in 3D are a probable cause for lowered oxidative stress, thus supporting the growing number of studies indicating that 2D cultures are an inappropriate, stressful method for cultivation of cells. Moreover the differences in the structural organization of the membrane coupled with the higher content of cholesterol and sphingomyelin, the major lipid components of the lipid rafts, in the plasma membranes of cells in 3D tissue-like environment, probably contribute to the differences in cell signalling.

The major difference between other 3D culturing systems and the cell derived in vivo-like three-dimensional matrices is the fact that their ECM is synthesized and organized naturally by the cells. In vivo, the extracellular matrix main functions are to present proper and specific 3D environment to cells and thus to define boundaries between different tissues. It provides the required elasticity and integrity during tissue and organ development, but is also being degraded and remodelled during both developmental processes and disease. Serving as an adhesive substrate, the ECM directs migratory cells and variations in its components concentrations may act as chemotactic gradient as well as differences in its mechanical properties can serve as durotactic gradient. The extracellular matrix also participates in the accumulation, storage, release and presentation of growth factors to the cells. The synthesis and immobilization of growth factors is spatially and temporally regulated by the ECM and the release of the incorporated ligands is also dependent upon appropriate cell-mediated forces, proteolitic degradation and proper presentation to cells. As a supportive structure, the ECM also participates in the reciprocal mechanical signalling. The transmittion of forces to and by the cells is regulated by their mechanical receptors - the integrins - and is manifested by changes in the intracellular signalling resulting in activation of the cytoskeleton machinery, growth factor production, proliferation rate alteration, etc. [83]. Among all these specific characteristic of the extracellular matrix probably the most important one is the fact that it is a dynamic structure, being constantly remodelled by cells in response to intrinsic signals, depending on the specific periods of organism's development or due to occurring diseases.

Currently cell-derived extracellular matrices are being applied with great success for in vitro investigation of developmental processes [84], tumor cell invasion and the role of the accompanying stroma cells [85], the mechanisms of fibrosis [86], studies of cellular migration [12, 87], as drug screening systems [88], for exploring the processes of wound healing [78] and numerous other applications. Despite the advantages of this model system, there are still a lot of considerable hindrances for its application in regenerative medicine and tissue engineering. Therefore numerous groups have undertaken a "reverse" approach towards meeting the requirements of modern medicine (see below).

\subsection{Decellularized tissues and organs as scaffolds for tissue engineering}

The need for organ transplantation in modern society far much exceeds the donor availability. Moreover the immunological incompatibility limits further the patient's possibilities for finding a matching donor for the subsequent transplantation. Even if all those criteria are met more often than not, patients are treated with powerful immunosupressants to reduce the chances of transplant rejection. 
The aspiring role of the ECM in governing the appropriate behaviour of a number of cell types led to the investigation of the properties and possible use in regenerative medicine of decellularized organs. The diversity of the extracellular matrix, its structure and micro-patterns suggest that specific cell types would overall "feel and perform better" if embedded in their corresponding natural matrix. There is a growing number of research to confirm this view. Culturing of hepatocytes as conventional monolayers, outside of their natural environment, results in loss of specific hepatocyte functions thus limiting the possibilities for their use in regenerative medicine. However, culturing of human hepatocytes in porcine liver-derived extracellular matrix supports albumin secretion and ammonia metabolism as well as restoration of hepatic transport activity [89]. Characteristic extracellular matrix is even required for the differentiation of human or murine embryonic stem cells to pneumocytes [90]. To date a number of ECM derived scaffolds have been described and investigated for use as organ reproducing systems, including heart-derived ECM [91], liver-derived ECM [92] and lungderived ECM [93].

The process of removal of cells from the organ and obtaining a cell-free scaffold is crucial for preserving the scaffold's qualities and usually includes several stages. Physical methods, involving freezing and thawing, mechanical agitation and sonication, could be considered non-harmful to the remaining biological scaffold and are usually combined with enzymatic and/or chemical methods, depending on the organ that is being decellularized. Treatment with exo-and endonucleases yields better results than physical methods, but is likely to affect the extracellular matrix as well. Harsh chemical treatment with acid or alkaline solutions, especially detergents, either ionic or non-ionic, is known to extract cells from the extracellular matrix, but also severely damages the remaining scaffold. Depending on the organ, its cellular content, overall lipid content, ECM biochemical composition, structure and complexity an appropriate method for decellularization has to be selected. More often than not a combination of methods is used to achieve better results. Successful removal of cells would ideally yield DNA- and cell debris-free extracellular matrix that is not affected or altered by the applied treatments, thus resulting in a minimal or even absent immunological reaction towards the allogenic or xenogenic extracellular matrix. Some protocols have already been developed and successfully used for producing biological scaffolds for heart, liver and lung [91-93].

Decellularization of organs would ultimately provide the most suitable scaffold for reconstruction of an organ - a natural extracellular matrix. Repopulation with cells of the obtained scaffold is a process which also poses some difficulties in organ reconstruction. Reintroduction of cells to such scaffold requires organ-specific types of cells as well as endothelial and epithelial cells for rebuilding of blood vessels, stem or progenitor type of cells to support future cell renewal in the organ and most of all, distinct methods for introduction of the appropriate cell types to their targeted environment. When possible, autologous cells are used since they are less likely to provoke immune response and be rejected. Such cells also present lesser chance of inducing cancer or the possible non-immune toxic reactions caused by immunosupressants [94]. Allogenic cells from matching donors could also be used for regenerative purposes when autologus cells cannot be harvested, or are terminally damaged. Although these cells are not derived from the patient, they have some advantages too. The required cell 
types can be derived from healthy individuals, characterized and maintained until needed, thereby providing the opportunity for faster therapy application. A well known example of such use of allogenic cells is provided by the mesenchymal bone marrow cells.

Often isolation of highly proliferating autologous cells from most organs is an almost impossible task. The use of embryonic stem cells could help solve this problem but the employment of human embryonic stem cells is still bounded up with heated moral debates. In addition it is a quite expensive technology, demanding proven methods for directed differentiation and extensive clinical trials. Therefore the use of adult cells with similar properties emerged as possible solution. Adult stem cells represent autologus cells which are multipotent and capable of self-renew. They have been known to exist in number of tissues like the gonads, intestine, skin and blood, and further data indicates their presence in adipose tissue [95], kidney [96], lung [97] and muscle [98]. In recent years, scientists have tried to identify specific markers to ease the isolation of adult stem cells, but such insignia are yet to be defined for most of them. Furthermore, it is currently evident that adult stem cells are localized in a specific extracellular environment - their niche. Signalling to and regulation of the self-renewal or differentiation processes appears to be tightly linked to the stem cell's niche, suggesting that micro-environmental cues may also be regulating cellular "stemness" [99]. A possible localization of different types of adult stem cells in niches in close proximity also exists [100, 101]. Thus, even though the specific localization of diverse types of adult stem cells have been identified, their isolation and further characterisation has proven to be an extremely difficult task. Label retention techniques and in vivo linage tracing as well as in vitro culturing and transplantation have yielded promising results and have aided the significant advancement in the field, but isolation of adult stem cells is still a major difficulty [99]. Despite the obstacles though, different types of adult stem cells have already found application in regenerative medicine [57, 102-104].

As pointed above, harvesting highly proliferating cells from healthy tissues has rendered a difficult task. Therefore researchers have sought to develop novel technologies that would aid obtaining and multiplication of such cells for further use in regenerative medicine. A promising technique developed just a few years ago is the induction of pluripotency in differentiated somatic cells. In 2006 Takahashi and Yamanaka introduced a method for inducing pluripotency in fibroblast cells by overexpression of four transcript factors - Oct-4, c-Myc, Sox 2 and Klf4 [105]. Their results showed that the timed overexpression of these factors is sufficient to convert fibroblasts to embryonic stem cell-like cells, termed induced pluripotent stem cells (iPSC), with many subsequent articles confirming their observations. The iPS cells are able to differentiate to any type of cell, just like embryonic stem cells, but since the progenitor cells are derived from the adult organism their use is liberated from the moral burdens concerning the use of embryonic stem cells. Research indicates that the genetic profile of good quality iPSC and embryonic stem cells is nearly identical, although some articles suggest that there may be some differences, probably attributed to different laboratory practices [106-108]. Further analysis of the whole genome have indeed found 71 differently methylated regions between iPSC and embryonic stem cells and 2,179 between iPSC and fibroblast [109], supporting the hypothesis that probably even though somatic cells are converted to pluripotent cells, there is still a "memory" preserved of the type of the donor cell. 
Even though there are a lot of difficulties with the production of iPS cells, already a plethora of articles has demonstrated the ability of iPS cells to differentiate to numerous types of cells, thus providing the ability to direct the in vitro differentiation of iPS cells to the required cell type for the specific therapy. Moreover significant results have been accomplished, namely the generation of adult mice from iPS cells [110] thus confirming the vast capabilities of such cells. Therapeutically, iPS cells have been used together with gene therapy to correct genetic defects in mice, with two studies already showing promising results in the treatment of different types of anaemia [111, 112]. Future efforts in the field of iPS cells though have to be made before a successful iPSC therapy for human patients becomes a reality - research is currently targeted towards the development of methods of pluripotency induction, not relying on viral transduction, therefore lowering the possibility of cancer induction by the transplanted iPS cells as well as improvement of the efficacy of reprogramming. Furthermore the remaining questions of whether the donor cells are completely reprogrammed or retain a "memory" of their differentiated state are still to be answered.

\section{Does a perfect 3D system exist?}

In vitro 3D models present the opportunity to investigate in depth the molecular mechanisms of the interactions between cells and the extracellular matrix. The increased number of research based on 3D scaffolds, mimicking specific physiological and developmental processes, as well as tissues, bridges the gap between laboratory investigation and unimpeded tissue regeneration. At the same time the increasing number of research has made it clear that variables such as cell source, the extracellular matrix's biochemical and mechanical properties, growth factor cues and developmental stage affect cell behavior and therapy outcome, imposing the need of even more careful reconsideration of yet the slightly interfering environmental factors for the successful outcome of regenerative therapies or tissue engineering.

Development of optimal bioengineered scaffolds requires indepth knowledge of physiology and understanding of cell-cell and cell-ECM interactions. The choice of inappropriate model system could have a negative effect on the study or the therapy outcome. Such an example could be any of the discussed 3D culturing systems. Sometimes even slight changes in experimental design like difference in cell count in collagen gels for example could lead to different migrational patterns [87] or as mentioned, the change of the substrate stiffness can induce different responses of cells to the environment [14]. Still, the obscured causes underlying such different outcomes are yet to be defined. Another example could be the inappropriate use of three-dimensional BME gels. In vivo basement membranes represent thin extracellular structures that underlie endothelial and epithelial structures. Basement membranes also surround muscle, fat and nerve cells. Moreover the composition and the amount of the basement membrane are specific and vary in different tissues and during particular stages of development. Even though introduction of cells to a 3D environment, based on basement membrane matrix, results in numerous morphological and physiological changes, there are a number of discrepancies to be considered and questions to be answered: 
- Contact with the basement membrane is not typical for all cell types, but a lot of cells express receptors for components of the basement membrane matrix. Therefore what would happen with cells that accidentally get caught in such unnatural environment? Would they become apoptotic or would they transform into malignant cells?

- Not all cell types respond to the basement membrane matrix and thus an optimization of the environment is required.

- Differences in basement membrane matrix composition during development imply that cells may respond to the matrix up to a certain stage. Acquisition and synchronization of cells of developmentally correct phases could be difficult and thus could inappropriately selected cells lead to activation of pathologic-like processes?

- The available basement membrane extract is of cancerous origin. Since tumor environment has been shown to be sufficient to promote desmoplastic differentiation [85] is it possible that contact of normal cells with cancerous cell-derived BME could induce their transformation?

All these discrepancies and unanswered questions do not make the BME gels unsuitable model systems, but stress the importance of experimental design. Hakkinen et al. have shown that normal fibroblasts respond unexpectedly in 3D BME gels - they acquire a rounded morphology with short protrusions, possibly for environmental sensing and do not migrate [12] behavior typical for non-malignant fibroblasts, thus supporting the notion that an adequate model should be chosen for each experimental design. Indeed BME gels provide scientists with a great tool to study malignant cell invasion through the basement membrane $[70,113]$, with numerous assays developed for investigation of the mechanisms of invasion. Moreover comparison between different three-dimensional scaffolds in terms of cell morphology, adhesion and migration and cytoskeletal organization concluded that indeed careful attention should be paid on the experimental design [12].

Use of other types of 3D gels faces researchers with similar problems. Neither collagen nor fibrin or hyaluronan gels incorporate other components of connective tissue. They are suitable for investigation of a specific response of a specific cell type towards distinctively modulated environments, but are still far away from the complex organization of the native extracellular environment. At the same time the provisional matrix of a blood clot is formed of disorganized fibrin mesh, just like the in vitro 3D gels. Cell-derived matrices on the other hand represent a complex, cell-organized structure based on cell-specific type of expressed matrix components. However, it is possible that in vitro generated cell derived matrices could also have different matrix composition, pore sizes, mechanical and biochemical characteristics than in vivo analogues because of the initially unnatural substrate cells were cultured on. Thus it is important of one to consider the specific characteristic of the tissue in vivo or the nature of the modeled process in order to be able to select an appropriate 3D matrix for in vitro research or for in vitro based preparation for therapies.

Besides looking at the ECM as a 3D structure one must not forget that in vivo cells do have a specific polarity that is usually lost during conventional 2D cultivation, and sometimes even in $3 \mathrm{D}$, if an inappropriate environment has been selected. Fibroblasts for example, lack an 
apical and basal organization in vivo. So when placed back in 3D environment after being cultured as 2D monolayers they tend to regain their typical in vivo morphology $[9,74,114]$, but the essence of the environment also has to be considered since it could provoke different morphology [12]. Moreover, both the mechanical and the structural state of the environment have to be taken into consideration. Recent studies have shown that gene expression patterns, fibroblast morphology, as well as organization of the extracellular environment differ not only between particular healthy tissues but also compared to tumor stroma [85, 115, 116]. Furthermore it is currently evident that cells do feel and respond in different ways to the environment - fibroblast cells differentiate to myofibroblasts due to changes in the substrate stiffness [86] and begin to remodel the extracellular matrix. Stem cells have the ability to differentiate to osteogenic, myoblastic and neuronal lineages based just on the change on substrate stiffness [13]. Tumor associated stroma alone was shown to be able to induce desmoplastic stroma fibroblast differentiation [85] as well as at later stages of tumor progression, to be more permissive for epithelial invasion [117]. Taking into consideration these differences it is not surprising that the compliance of the substrate and its topography and mechanical features can control cellular behavior. Therefore the transplantation of cells into bioengineered scaffolds for the purposes of regenerative medicine or tissue engineering has to be precisely assessed and executed, since even the small differences in the substrate's composition, organization or stiffness have the potential to alter the donor cells gene expression $[85,118]$ or to promote tumorigenic transformation [119].

Despite the drawbacks revealed about each type of bioengineered scaffolds they have already found use in regenerative medicine. Fibrin and collagen grafts for example were among the first used in burn patients for skin reconstruction. Study by Chua et al. has shown that use of skin tissue constructs has reduced mortality in patients with $60 \%$ of total body area burns from $100 \%$ in 1952 to $41,4 \%$ in 2003 [120]. The advance in the skin tissue regeneration is accentuated by the high number of currently available off-the-shelf bioengineered skin grafts [46], that could be grouped by several criteria:

- Biomaterial - biological (autologus, allogenic and xenogenic) and synthetic (biodegradable, non-biodegradable)

- Duration of the graft - permanent, semi-permanent, temporary

- Composition regarding cell content - cellularized or acellular

- Anatomical structure - epidermal, dermal, composite

Together with this immense success in the field of burn wounds and the increase in survival rate though, patients are faced with new obstacles. More often than not wounds in surviving patients develop severe fibrosis after healing. The resulting hypertrophic scars present a major discomfort in survivor's life by possibly limiting the range of motion of joints, also resulting in impaired thermal regulation and not at the least a disturbed visual appearance [121].

In spite of the advances made in skin tissue engineering and without undervaluing the accomplished results there is still a lot of progress to be made towards the prefect reconstruction of the skin. There still isn't an available option that makes possible the regeneration of 
hair follicles and sebatious glands, thus allowing for rehabilitation of thermal regulation. Even though the perfect skin graft is still to be created, the available skin models have made a significant impact in in vitro studies regarding skin biology and skin disease progression: engineered skin of different aspects helps in producing more relevant results than 2D cultures, thus leading to reduced use of animals for experimentation. As discussed earlier investigation of cell-cell and cell-extracellular matrix interactions has evolved rapidly due to the use of skin tissue-mimicking models. Wound healing, skin contraction [122] and angiogenesis are all fields benefiting from the use of 3D skin equivalents. Investigation of skin diseases such as melanoma invasion [123, 124], psoriasis [125] and skin blistering disorders [126] is also making significant progress based on engineered skin.

Besides skin tissue engineering biological scaffolds have found use in other sections of tissue engineering. Promising results are obtained with decellularization and recellularization of heart, liver and lungs. After recellularization of a hearth-derived ECM, a macroscopic contraction and pump function of the newly generated heart were observed in vitro [91] - indeed a promising result for the generation of a biological artificial heart. Recellularization of liver [92] and lungs [127] has gone even further and transplantation of the newly formed organs back to animals showed that they are able to sustain their natural functions. Probably one of the highest successes of tissue engineering for regenerative medicine is the successful transplant of trachea in a 30-year old patient [128] based on decellularized tracheal scaffold, reseeded with autologus epithelial and mesenchymal cells thus reducing the possibility of organ rejection and the need of strong immunosupressants. Despite the great success in the field the major disadvantage of these scaffolds still persists - the need of a donor organ for their preparation. Nevertheless the first successful transplantation of entirely bioengineered constructs, a completely new tracheobronchial tube in a patient suffering from tracheal cancer, is already a reality, partly due to the virtue to three-dimensional model systems [129].

Based on the review of decades of research it is currently evident that a perfect 3D system able to fulfill all scientific and medicinal requirements does not exist. On the contrary - a careful and differential approach towards appropriate 3D system selection, experimental or therapeutical design and data interpretation is required for every particular case (Figure 2). Such approach would contribute to the optimal outcome of the specific therapy or the distinct experiment thus leading the way to unimpeded regenerative medicine.

\section{Conclusion}

The evolution of the cell culturing method has led to the sophistication of culturing procedures with one main goal - to approximate in vitro conditions to in vivo. The advancement of threedimensional culturing models has allowed for an in depth understanding of cellular behavior and especially the role of the cell's environment has emerged as an important modulator of cellular functions. Current data has made it clear that future development and use of superior three-dimensional cultures should focus not just on the dimensionality of the environment per se, but mainly on its characteristics - biochemical composition, production source and possible 
unwanted cell-cueing signals, mechanical properties, etc. thus making 3D culturing, along with regenerative medicine multidisciplinary fields. However, as science advances, more and more questions emerge and await answers in order to confirm the long term safety of the applied methods for human therapy.

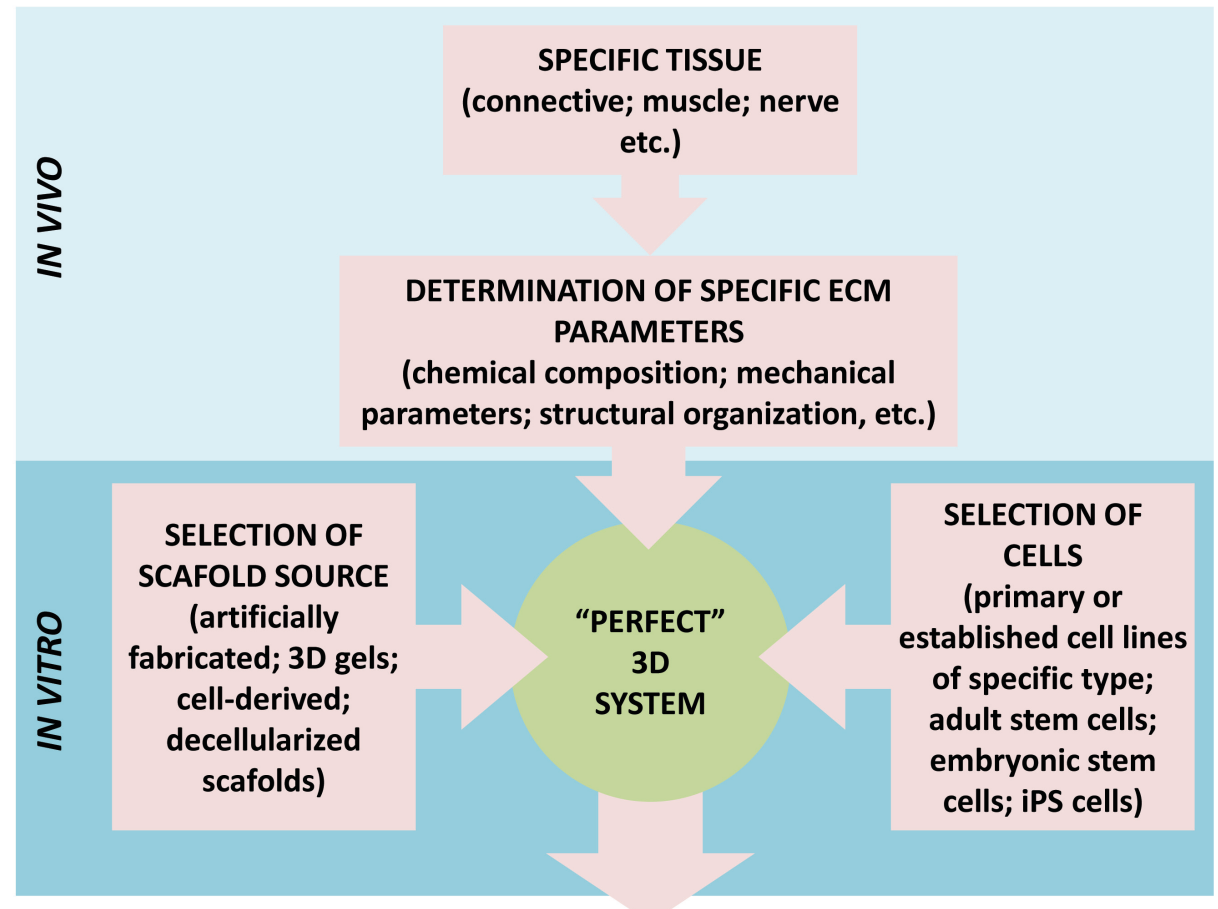

RESEARCH AND/OR MEDICAL APPLICATION

Figure 2. General strategy for design of a "perfect" 3D system. The specific set of ECM characteristics, typical for selected normal tissue can be determined. Based on these studies, a scaffold with matching parameters and suitable cells can be identified and combined in vitro for development of particular, tailored made 3D culture, meeting the needs of a specific research or specific therapy.

During the resolution of the moral issues concerning the use of human embryonic stem cells or donor organs the remaining fields continue to advance rapidly. It appears that isolation of autologus cells, their expansion in vitro and probably stimulation to produce extracellular matrix with the required dimensionality, biochemical and mechanical properties would represent the optimal tissue engineered scaffold for tissue and organ reconstruction. Further advance in the field of regenerative medicine and tissue engineering would possibly see the development of 4D model systems, incorporating time as the forth dimension. Such models would represent not just single stages of organ development or disease, but the chronology of 
their maturation/progression, thus providing an opportunity to transfer developmental processes to in vitro conditions.

\section{Acknowledgements}

This publication is supported with funding form Grant № ДДВУ02/9 by the National Science Fund

\section{Author details}

Aleksandar Evangelatov and Roumen Pankov

Department of Cytology, Histology and Embryology, Sofia University "St. Kliment Ohridski", Sofia, Bulgaria

\section{References}

[1] Hamburger V. Wilhelm Roux: visionary with a blind spot. J Hist Biol 1997;30(2) 229-38.

[2] Carrel A. On the Permanent Life of Tissues Outside of the Organism. J Exp Med 1912;15(5) 516-28.

[3] Eagle H. Nutrition needs of mammalian cells in tissue culture. Science 1955;122(3168) 501-14.

[4] Scherer WF, Syverton JT, Gey GO. Studies on the propagation in vitro of poliomyelitis viruses. IV. Viral multiplication in a stable strain of human malignant epithelial cells (strain HeLa) derived from an epidermoid carcinoma of the cervix. J Exp Med 1953;97(5) 695-710.

[5] Martin GR. Isolation of a pluripotent cell line from early mouse embryos cultured in medium conditioned by teratocarcinoma stem cells. Proc Natl Acad Sci U S A 1981;78(12) 7634-8.

[6] Evans MJ, Kaufman MH. Establishment in culture of pluripotential cells from mouse embryos. Nature 1981;292(5819) 154-6.

[7] Thomson JA, Itskovitz-Eldor J, Shapiro SS, Waknitz MA, Swiergiel JJ, Marshall VS, et al. Embryonic stem cell lines derived from human blastocysts. Science 1998;282(5391) 1145-7. 
[8] Damianova R, Stefanova N, Cukierman E, Momchilova A, Pankov R. Three-dimensional matrix induces sustained activation of ERK1/2 via Src/Ras/Raf signaling pathway. Cell Biol Int 2008;32(2) 229-34.

[9] Cukierman E, Pankov R, Stevens DR, Yamada KM. Taking cell-matrix adhesions to the third dimension. Science 2001;294(5547) 1708-12.

[10] Geiger B, Bershadsky A, Pankov R, Yamada KM. Transmembrane crosstalk between the extracellular matrix--cytoskeleton crosstalk. Nat Rev Mol Cell Biol 2001;2(11) 793-805.

[11] Friedl P, Brocker EB. The biology of cell locomotion within three-dimensional extracellular matrix. Cell Mol Life Sci 2000;57(1) 41-64.

[12] Hakkinen KM, Harunaga JS, Doyle AD, Yamada KM. Direct comparisons of the morphology, migration, cell adhesions, and actin cytoskeleton of fibroblasts in four different three-dimensional extracellular matrices. Tissue Eng Part A 2011;17(5-6) 713-24.

[13] Engler AJ, Sen S, Sweeney HL, Discher DE. Matrix elasticity directs stem cell lineage specification. Cell 2006;126(4) 677-89.

[14] Discher DE, Janmey P, Wang YL. Tissue cells feel and respond to the stiffness of their substrate. Science 2005;310(5751) 1139-43.

[15] Zamir E, Katz BZ, Aota S, Yamada KM, Geiger B, Kam Z. Molecular diversity of cellmatrix adhesions. J Cell Sci 1999;112 ( Pt 11) 1655-69.

[16] Barcellos-Hoff MH, Aggeler J, Ram TG, Bissell MJ. Functional differentiation and alveolar morphogenesis of primary mammary cultures on reconstituted basement membrane. Development 1989;105(2) 223-35.

[17] Elsdale T, Bard J. Collagen substrata for studies on cell behavior. J Cell Biol 1972;54(3) 626-37.

[18] Trinh LA, Stainier DY. Fibronectin regulates epithelial organization during myocardial migration in zebrafish. Dev Cell 2004;6(3) 371-82.

[19] Ryan MC, Lee K, Miyashita Y, Carter WG. Targeted disruption of the LAMA3 gene in mice reveals abnormalities in survival and late stage differentiation of epithelial cells. J Cell Biol 1999;145(6) 1309-23.

[20] Liu X, Wu H, Byrne M, Jeffrey J, Krane S, Jaenisch R. A targeted mutation at the known collagenase cleavage site in mouse type I collagen impairs tissue remodeling. J Cell Biol 1995;130(1) 227-37.

[21] Wenstrup RJ, Florer JB, Brunskill EW, Bell SM, Chervoneva I, Birk DE. Type V collagen controls the initiation of collagen fibril assembly. J Biol Chem 2004;279(51) 53331-7. 
[22] Wagenseil JE, Mecham RP. Vascular extracellular matrix and arterial mechanics. Physiol Rev 2009;89(3) 957-89.

[23] Arikawa-Hirasawa E, Watanabe H, Takami H, Hassell JR, Yamada Y. Perlecan is essential for cartilage and cephalic development. Nat Genet 1999;23(3) 354-8.

[24] Watanabe H, Kimata K, Line S, Strong D, Gao LY, Kozak CA, et al. Mouse cartilage matrix deficiency (cmd) caused by a 7 bp deletion in the aggrecan gene. Nat Genet 1994;7(2) 154-7.

[25] Martin-Belmonte F, Gassama A, Datta A, Yu W, Rescher U, Gerke V, et al. PTENmediated apical segregation of phosphoinositides controls epithelial morphogenesis through Cdc42. Cell 2007;128(2) 383-97.

[26] Kesavan G, Sand FW, Greiner TU, Johansson JK, Kobberup S, Wu X, et al. Cdc42mediated tubulogenesis controls cell specification. Cell 2009;139(4) 791-801.

[27] Burridge K TS. Editorial overview: Cell-to-cell contact and extracellular matrix. Current Opinion in Cell Biology 2001(13) 525-8.

[28] Hynes RO. Cell adhesion: old and new questions. Trends Cell Biol 1999;9(12) M33-7.

[29] Inman JL, Bissell MJ. Apical polarity in three-dimensional culture systems: where to now? J Biol 2010;9(1) 2.

[30] Persidis A. Tissue engineering. Nat Biotechnol 1999;17(5) 508-10.

[31] Liu X, Won Y, Ma PX. Porogen-induced surface modification of nano-fibrous poly(Llactic acid) scaffolds for tissue engineering. Biomaterials 2006;27(21) 3980-7.

[32] Liu X, Smith LA, Hu J, Ma PX. Biomimetic nanofibrous gelatin/apatite composite scaffolds for bone tissue engineering. Biomaterials 2009;30(12) 2252-8.

[33] Ozkan S, Kalyon DM, Yu X, McKelvey CA, Lowinger M. Multifunctional protein-encapsulated polycaprolactone scaffolds: fabrication and in vitro assessment for tissue engineering. Biomaterials 2009;30(26) 4336-47.

[34] Sun W, Lal P. Recent development on computer aided tissue engineering--a review. Comput Methods Programs Biomed 2002;67(2) 85-103.

[35] Zhang S, Zhao X. Design of molecular biological materials using peptide motifs. Journal of Materials Chemistry 2004;14(14) 2082-6.

[36] Li D, Xia Y. Electrospinning of Nanofibers: Reinventing the Wheel? Advanced Materials 2004;16(14) 1151-70.

[37] Gordon MK, Hahn RA. Collagens. Cell Tissue Res 2010;339(1) 247-57.

[38] Schor SL, Schor AM, Winn B, Rushton G. The use of three-dimensional collagen gels for the study of tumour cell invasion in vitro: experimental parameters influencing cell migration into the gel matrix. Int J Cancer 1982;29(1) 57-62. 
[39] Grinnell F. Fibroblast-collagen-matrix contraction: growth-factor signalling and mechanical loading. Trends Cell Biol 2000;10(9) 362-5.

[40] Petersen OW, Ronnov-Jessen L, Howlett AR, Bissell MJ. Interaction with basement membrane serves to rapidly distinguish growth and differentiation pattern of normal and malignant human breast epithelial cells. Proc Natl Acad Sci U S A 1992;89(19) 9064-8.

[41] Koutsilieris M, Sourla A, Pelletier G, Doillon CJ. Three-dimensional type I collagen gel system for the study of osteoblastic metastases produced by metastatic prostate cancer. J Bone Miner Res 1994;9(11) 1823-32.

[42] Parizi M, Howard EW, Tomasek JJ. Regulation of LPA-promoted myofibroblast contraction: role of Rho, myosin light chain kinase, and myosin light chain phosphatase. Exp Cell Res 2000;254(2) 210-20.

[43] Vaughan MB, Howard EW, Tomasek JJ. Transforming growth factor-beta1 promotes the morphological and functional differentiation of the myofibroblast. Exp Cell Res 2000;257(1) 180-9.

[44] Halliday NL, Tomasek JJ. Mechanical properties of the extracellular matrix influence fibronectin fibril assembly in vitro. Exp Cell Res 1995;217(1) 109-17.

[45] Bell E, Ehrlich HP, Buttle DJ, Nakatsuji T. Living tissue formed in vitro and accepted as skin-equivalent tissue of full thickness. Science 1981;211(4486) 1052-4.

[46] Shevchenko RV, James SL, James SE. A review of tissue-engineered skin bioconstructs available for skin reconstruction. J R Soc Interface 2010;7(43) 229-58.

[47] Even-Ram S. Fibrin gel model for assessment of cellular contractility. Methods Mol Biol 2009;522 251-9.

[48] Horch RE, Bannasch H, Kopp J, Andree C, Stark GB. Single-cell suspensions of cultured human keratinocytes in fibrin-glue reconstitute the epidermis. Cell Transplant $1998 ; 7(3)$ 309-17.

[49] Shaikh FM, Callanan A, Kavanagh EG, Burke PE, Grace PA, McGloughlin TM. Fibrin: a natural biodegradable scaffold in vascular tissue engineering. Cells Tissues Organs 2008;188(4) 333-46.

[50] Yuan Ye K, Sullivan KE, Black LD. Encapsulation of cardiomyocytes in a fibrin hydrogel for cardiac tissue engineering. J Vis Exp 2011(55).

[51] Chien CS, Ho HO, Liang YC, Ko PH, Sheu MT, Chen CH. Incorporation of exudates of human platelet-rich fibrin gel in biodegradable fibrin scaffolds for tissue engineering of cartilage. J Biomed Mater Res B Appl Biomater 2012;100(4) 948-55.

[52] Fraser JR, Laurent TC, Laurent UB. Hyaluronan: its nature, distribution, functions and turnover. J Intern Med 1997;242(1) 27-33. 
[53] Kang JY, Chung CW, Sung JH, Park BS, Choi JY, Lee SJ, et al. Novel porous matrix of hyaluronic acid for the three-dimensional culture of chondrocytes. Int J Pharm 2009;369(1-2) 114-20.

[54] Amini AA, Nair LS. Injectable hydrogels for bone and cartilage repair. Biomed Mater 2012;7(2) 024105.

[55] Pavesio A, Abatangelo G, Borrione A, Brocchetta D, Hollander AP, Kon E, et al. Hyaluronan-based scaffolds (Hyalograft $C$ ) in the treatment of knee cartilage defects: preliminary clinical findings. Novartis Found Symp 2003;249 203-17; discussion 29-33, $34-8,39-41$.

[56] Kon E, Filardo G, Tschon M, Fini M, Giavaresi G, Marchesini Reggiani L, et al. TISSUE ENGINEERING FOR TOTAL MENISCAL SUBSTITUTION. Animal study in sheep model: results at 12 months. Tissue Eng Part A 2012.

[57] Stok KS, Lisignoli G, Cristino S, Facchini A, Muller R. Mechano-functional assessment of human mesenchymal stem cells grown in three-dimensional hyaluronanbased scaffolds for cartilage tissue engineering. J Biomed Mater Res A 2010;93(1) $37-45$.

[58] Yuan Ye K, Sullivan KE, Black LD. Encapsulation of cardiomyocytes in a fibrin hydrogel for cardiac tissue engineering. J Vis Exp (55).

[59] Skardal A, Smith L, Bharadwaj S, Atala A, Soker S, Zhang Y. Tissue specific synthetic ECM hydrogels for 3-D in vitro maintenance of hepatocyte function. Biomaterials 2012;33(18) 4565-75.

[60] Young DA, Christman KL. Injectable biomaterials for adipose tissue engineering. Biomed Mater 2012;7(2) 024104.

[61] Pakulska MM, Ballios BG, Shoichet MS. Injectable hydrogels for central nervous system therapy. Biomed Mater 2012;7(2) 024101.

[62] Kleinman HK, McGarvey ML, Liotta LA, Robey PG, Tryggvason K, Martin GR. Isolation and characterization of type IV procollagen, laminin, and heparan sulfate proteoglycan from the EHS sarcoma. Biochemistry 1982;21(24) 6188-93.

[63] Hadley MA, Byers SW, Suarez-Quian CA, Kleinman HK, Dym M. Extracellular matrix regulates Sertoli cell differentiation, testicular cord formation, and germ cell development in vitro. J Cell Biol 1985;101(4) 1511-22.

[64] Carey DJ, Todd MS, Rafferty CM. Schwann cell myelination: induction by exogenous basement membrane-like extracellular matrix. J Cell Biol 1986;102(6) 2254-63.

[65] Li ML, Aggeler J, Farson DA, Hatier C, Hassell J, Bissell MJ. Influence of a reconstituted basement membrane and its components on casein gene expression and secretion in mouse mammary epithelial cells. Proc Natl Acad Sci U S A 1987;84(1) 136-40. 
[66] Kubota Y, Kleinman HK, Martin GR, Lawley TJ. Role of laminin and basement membrane in the morphological differentiation of human endothelial cells into capillarylike structures. J Cell Biol 1988;107(4) 1589-98.

[67] Benelli R, Albini A. In vitro models of angiogenesis: the use of Matrigel. Int J Biol Markers 1999;14(4) 243-6.

[68] Kramer RH, Bensch KG, Wong J. Invasion of reconstituted basement membrane matrix by metastatic human tumor cells. Cancer Res 1986;46(4 Pt 2) 1980-9.

[69] Albini A, Iwamoto Y, Kleinman HK, Martin GR, Aaronson SA, Kozlowski JM, et al. A rapid in vitro assay for quantitating the invasive potential of tumor cells. Cancer Res 1987;47(12) 3239-45.

[70] Albini A, Benelli R, Noonan DM, Brigati C. The "chemoinvasion assay": a tool to study tumor and endothelial cell invasion of basement membranes. Int J Dev Biol 2004;48(5-6) 563-71.

[71] Xu C, Inokuma MS, Denham J, Golds K, Kundu P, Gold JD, et al. Feeder-free growth of undifferentiated human embryonic stem cells. Nat Biotechnol 2001;19(10) 971-4.

[72] LeBleu VS, Macdonald B, Kalluri R. Structure and function of basement membranes. Exp Biol Med (Maywood) 2007;232(9) 1121-9.

[73] Hakala H, Rajala K, Ojala M, Panula S, Areva S, Kellomaki M, et al. Comparison of biomaterials and extracellular matrices as a culture platform for multiple, independently derived human embryonic stem cell lines. Tissue Eng Part A 2009;15(7) 1775-85.

[74] Grinnell F. Fibroblast biology in three-dimensional collagen matrices. Trends Cell Biol 2003;13(5) 264-9.

[75] Beacham DA, Amatangelo MD, Cukierman E. Preparation of extracellular matrices produced by cultured and primary fibroblasts. Curr Protoc Cell Biol 2007; Chapter 10 Unit 109.

[76] Grinnell F, Fukamizu H, Pawelek P, Nakagawa S. Collagen processing, crosslinking, and fibril bundle assembly in matrix produced by fibroblasts in long-term cultures supplemented with ascorbic acid. Exp Cell Res 1989;181(2) 483-91.

[77] Clark RA, McCoy GA, Folkvord JM, McPherson JM. TGF-beta 1 stimulates cultured human fibroblasts to proliferate and produce tissue-like fibroplasia: a fibronectin matrix-dependent event. J Cell Physiol 1997;170(1) 69-80.

[78] Ohgoda O, Sakai A, Koga H, Kanai K, Miyazaki T, Niwano Y. Fibroblast-migration in a wound model of ascorbic acid-supplemented three-dimensional culture system: the effects of cytokines and malotilate, a new wound healing stimulant, on cell-migration. J Dermatol Sci 1998;17(2) 123-31.

[79] Ahlfors JE, Billiar KL. Biomechanical and biochemical characteristics of a human fibroblast-produced and remodeled matrix. Biomaterials 2007;28(13) 2183-91. 
[80] Stefanova N, Staneva G, Petkova D, Lupanova T, Pankov R, Momchilova A. Cell culturing in a three-dimensional matrix affects the localization and properties of plasma membrane cholesterol. Cell Biol Int 2009;33(10) 1079-86.

[81] Lupanova T, Stefanova N, Petkova D, Staneva G, Jordanova A, Koumanov K, et al. Alterations in the content and physiological role of sphingomyelin in plasma membranes of cells cultured in three-dimensional matrix. Mol Cell Biochem 2010;340(1-2) 215-22.

[82] Staneva G, Lupanova T, Chachaty C, Petkova D, Koumanov K, Pankov R, et al. Structural organization of plasma membrane lipids isolated from cells cultured as a monolayer and in tissue-like conditions. J Colloid Interface Sci 2011;359(1) 202-9.

[83] Rozario T, DeSimone DW. The extracellular matrix in development and morphogenesis: a dynamic view. Dev Biol 2010;341(1) 126-40.

[84] Onodera T, Sakai T, Hsu JC, Matsumoto K, Chiorini JA, Yamada KM. Btbd7 regulates epithelial cell dynamics and branching morphogenesis. Science 2010;329(5991) 562-5.

[85] Amatangelo MD, Bassi DE, Klein-Szanto AJ, Cukierman E. Stroma-derived three-dimensional matrices are necessary and sufficient to promote desmoplastic differentiation of normal fibroblasts. Am J Pathol 2005;167(2) 475-88.

[86] Hinz B. Tissue stiffness, latent TGF-beta1 activation, and mechanical signal transduction: implications for the pathogenesis and treatment of fibrosis. Curr Rheumatol Rep 2009;11(2) 120-6.

[87] Friedl P. Prespecification and plasticity: shifting mechanisms of cell migration. Curr Opin Cell Biol 2004;16(1) 14-23.

[88] Cukierman E, Bassi D. The mesenchymal tumor microenvironment: A drug resistant niche. Cell Adh Migr 2012;6(3) 285-96.

[89] Sellaro TL, Ranade A, Faulk DM, McCabe GP, Dorko K, Badylak SF, et al. Maintenance of human hepatocyte function in vitro by liver-derived extracellular matrix gels. Tissue Eng Part A 2010;16(3) 1075-82.

[90] Lin YM, Zhang A, Rippon HJ, Bismarck A, Bishop AE. Tissue engineering of lung: the effect of extracellular matrix on the differentiation of embryonic stem cells to pneumocytes. Tissue Eng Part A 2010;16(5) 1515-26.

[91] Ott HC, Matthiesen TS, Goh SK, Black LD, Kren SM, Netoff TI, et al. Perfusion-decellularized matrix: using nature's platform to engineer a bioartificial heart. Nat Med 2008;14(2) 213-21.

[92] Uygun BE, Soto-Gutierrez A, Yagi H, Izamis ML, Guzzardi MA, Shulman C, et al. Organ reengineering through development of a transplantable recellularized liver graft using decellularized liver matrix. Nat Med 2010;16(7) 814-20. 
[93] Petersen TH, Calle EA, Zhao L, Lee EJ, Gui L, Raredon MB, et al. Tissue-engineered lungs for in vivo implantation. Science 2010;329(5991) 538-41.

[94] Benoit E, O'Donnell Jr TF, Patel AN. Safety and efficacy of autologous cell therapy in critical limb ischemia: a systematic review of the literature. Cell Transplant 2012.

[95] Baer PC, Geiger H. Adipose-derived mesenchymal stromal/stem cells: tissue localization, characterization, and heterogeneity. Stem Cells Int 2012;2012 812693.

[96] Oliver JA, Maarouf O, Cheema FH, Martens TP, Al-Awqati Q. The renal papilla is a niche for adult kidney stem cells. J Clin Invest 2004;114(6) 795-804.

[97] Kim CF, Jackson EL, Woolfenden AE, Lawrence S, Babar I, Vogel S, et al. Identification of bronchioalveolar stem cells in normal lung and lung cancer. Cell 2005;121(6) 823-35.

[98] Collins CA, Olsen I, Zammit PS, Heslop L, Petrie A, Partridge TA, et al. Stem cell function, self-renewal, and behavioral heterogeneity of cells from the adult muscle satellite cell niche. Cell 2005;122(2) 289-301.

[99] Snippert HJ, Clevers H. Tracking adult stem cells. EMBO Rep 2011;12(2) 113-22.

[100] Levy V, Lindon C, Harfe BD, Morgan BA. Distinct stem cell populations regenerate the follicle and interfollicular epidermis. Dev Cell 2005;9(6) 855-61.

[101] Sommer L. Checkpoints of melanocyte stem cell development. Sci STKE 2005;2005(298) pe42.

[102] Gir P, Oni G, Brown SA, Mojallal A, Rohrich RJ. Human adipose stem cells: current clinical applications. Plast Reconstr Surg 2012;129(6) 1277-90.

[103] Martin U. Methods for studying stem cells: adult stem cells for lung repair. Methods $2008 ; 45(2)$ 121-32.

[104] Roche R, Hoareau L, Mounet F, Festy F. Adult stem cells for cardiovascular diseases: the adipose tissue potential. Expert Opin Biol Ther 2007;7(6) 791-8.

[105] Takahashi K, Yamanaka S. Induction of pluripotent stem cells from mouse embryonic and adult fibroblast cultures by defined factors. Cell 2006;126(4) 663-76.

[106] Chin MH, Mason MJ, Xie W, Volinia S, Singer M, Peterson C, et al. Induced pluripotent stem cells and embryonic stem cells are distinguished by gene expression signatures. Cell Stem Cell 2009;5(1) 111-23.

[107] Guenther MG, Frampton GM, Soldner F, Hockemeyer D, Mitalipova M, Jaenisch R, et al. Chromatin structure and gene expression programs of human embryonic and induced pluripotent stem cells. Cell Stem Cell 2010;7(2) 249-57.

[108] Newman AM, Cooper JB. Lab-specific gene expression signatures in pluripotent stem cells. Cell Stem Cell 2010;7(2) 258-62. 
[109] Doi A, Park IH, Wen B, Murakami P, Aryee MJ, Irizarry R, et al. Differential methylation of tissue- and cancer-specific CPG island shores distinguishes human induced pluripotent stem cells, embryonic stem cells and fibroblasts. Nat Genet 2009;41(12) 1350-3.

[110] Boland MJ, Hazen JL, Nazor KL, Rodriguez AR, Gifford W, Martin G, et al. Adult mice generated from induced pluripotent stem cells. Nature 2009;461(7260) 91-4.

[111] Raya A, Rodriguez-Piza I, Guenechea G, Vassena R, Navarro S, Barrero MJ, et al. Disease-corrected haematopoietic progenitors from Fanconi anaemia induced pluripotent stem cells. Nature 2009;460(7251) 53-9.

[112] Hanna J, Wernig M, Markoulaki S, Sun CW, Meissner A, Cassady JP, et al. Treatment of sickle cell anemia mouse model with iPS cells generated from autologous skin. Science 2007;318(5858) 1920-3.

[113] Friedl P, Wolf K. Tube travel: the role of proteases in individual and collective cancer cell invasion. Cancer Res 2008;68(18) 7247-9.

[114] Beningo KA, Dembo M, Wang YL. Responses of fibroblasts to anchorage of dorsal extracellular matrix receptors. Proc Natl Acad Sci U S A 2004;101(52) 18024-9.

[115] Kalluri R, Zeisberg M. Fibroblasts in cancer. Nat Rev Cancer 2006;6(5) 392-401.

[116] Singer CF, Gschwantler-Kaulich D, Fink-Retter A, Haas C, Hudelist G, Czerwenka K, et al. Differential gene expression profile in breast cancer-derived stromal fibroblasts. Breast Cancer Res Treat 2008;110(2) 273-81.

[117] Tuxhorn JA, Ayala GE, Rowley DR. Reactive stroma in prostate cancer progression. J Urol 2001;166(6) 2472-83.

[118] Sato M, Sato T, Kojima N, Imai K, Higashi N, Wang DR, et al. 3-D structure of extracellular matrix regulates gene expression in cultured hepatic stellate cells to induce process elongation. Comp Hepatol 2004;3 Suppl 1 S4.

[119] Paszek MJ, Zahir N, Johnson KR, Lakins JN, Rozenberg GI, Gefen A, et al. Tensional homeostasis and the malignant phenotype. Cancer Cell 2005;8(3) 241-54.

[120] Chua A, Song C, Chai A, Kong S, Tan KC. Use of skin allograft and its donation rate in Singapore: an 11-year retrospective review for burns treatment. Transplant Proc 2007;39(5) 1314-6.

[121] Goel A, Shrivastava P. Post-burn scars and scar contractures. Indian J Plast Surg 2010;43(Suppl) S63-71.

[122] Harrison CA, Gossiel F, Layton CM, Bullock AJ, Johnson T, Blumsohn A, et al. Use of an in vitro model of tissue-engineered skin to investigate the mechanism of skin graft contraction. Tissue Eng 2006;12(11) 3119-33. 
[123] Meier F, Nesbit M, Hsu MY, Martin B, Van Belle P, Elder DE, et al. Human melanoma progression in skin reconstructs : biological significance of bFGF. Am J Pathol 2000;156(1) 193-200.

[124] Eves P, Layton C, Hedley S, Dawson RA, Wagner M, Morandini R, et al. Characterization of an in vitro model of human melanoma invasion based on reconstructed human skin. Br J Dermatol 2000;142(2) 210-22.

[125] Barker CL, McHale MT, Gillies AK, Waller J, Pearce DM, Osborne J, et al. The development and characterization of an in vitro model of psoriasis. J Invest Dermatol 2004;123(5) 892-901.

[126] Ferrari S, Pellegrini G, Matsui T, Mavilio F, De Luca M. Gene therapy in combination with tissue engineering to treat epidermolysis bullosa. Expert Opin Biol Ther 2006;6(4) 367-78.

[127] Petersen TH, Calle EA, Zhao L, Lee EJ, Gui L, Raredon MB, et al. Tissue-engineered lungs for in vivo implantation. Science;329(5991) 538-41.

[128] Macchiarini P, Jungebluth P, Go T, Asnaghi MA, Rees LE, Cogan TA, et al. Clinical transplantation of a tissue-engineered airway. Lancet 2008;372(9655) 2023-30.

[129] Jungebluth P, Alici E, Baiguera S, Le Blanc K, Blomberg P, Bozoky B, et al. Tracheobronchial transplantation with a stem-cell-seeded bioartificial nanocomposite: a proof-of-concept study. Lancet 2011;378(9808) 1997-2004. 